\title{
SISTEMAS NEURAIS HÍBRIDOS PARA RECONHECIMENTO DE PADRÕES EM NARIZES ARTIFICIAIS
}

\author{
Cleber Zanchettin* \\ cz@cin.ufpe.br
}

\author{
Teresa Bernarda Ludermir* \\ tbl@cin.ufpe.br
}

\author{
${ }^{*}$ Centro de Informática \\ Universidade Federal de Pernambuco - UFPE \\ Caixa Postal 7851 - 50.732-970 \\ Recife - PE - Brasil
}

\begin{abstract}
This work investigates the use of Hybrid Intelligent Systems in the pattern recognition system of an artificial nose. The connectionist approaches Multi-Layer Perceptron and Time Delay Neural Networks; and the hybrid approaches Feature-weighted Detector and Evolving Neural Fuzzy Networks were investigated. A wavelet filter as preprocessing method of odors signals is evaluated. The signals generated by an artificial nose, composed by an array of conducting polymer sensors, exposed to two different odor databases.
\end{abstract}

KEYWORDS: Hybrid Intelligent Systems, Artificial Neural Networks, Artificial Nose.

\section{RESUMO}

Este trabalho investiga a utilização de Sistemas Híbridos Inteligentes no sistema de reconhecimento de padrões de um nariz artificial. São abordadas as arquiteturas conexionistas Multi-Layer Perceptron e Time Delay Neural Network; e as arquiteturas híbridas Feature-weighted Detector e Evolving Fuzzy Neural Networks. Além dos classificadores, um filtro Wavelet é avaliado como método de pré-processamento para os sinais de odores. Foram analisados sinais gerados por um nariz artificial, composto por um conjunto de sensores de polímeros condutores, exposto a duas bases de odores distintas.

PALAVRAS-CHAVE: Sistemas Híbridos Inteligentes, Redes Neurais Artificiais, Nariz Artificial.

\section{INTRODUÇÃO}

Os narizes artificiais são dispositivos eletrônicos capazes de detectar e classificar odores, vapores e gases automaticamente. Todavia, apesar da pesquisa e desenvolvimento dos narizes artificiais serem inspirados no sistema olfativo biológico, sua implementação, não se aproxima nem da complexidade ou de muitas das funcionalidades de seu equivalente biológico. Atualmente, estes dispositivos consistem de um sistema sensor e um sistema de reconhecimento de padrões.

Nos narizes artificiais o processo de reconhecimento e classificação do odor se inicia no sistema sensor, responsável pela captação ou medição dos estímulos odorantes no ambiente frente à sensibilidade de seus sensores. Cada composto odorante apresentado ao sistema sensor do nariz artificial produz um estímulo característico, frente aos sensores de detecção. Após a captação destes estímulos ou padrões de odores, e antes que estes sinais sejam apresentados ao sistema de reconhecimento de padrões, realiza-se uma etapa de préprocessamento nos dados.

A fase de pré-processamento nos padrões de odores tem como finalidade a análise dos dados, extração dos descritores, correção de distorções, normalização e remoção/diminuição dos erros de leitura. Pré-processados, os padrões de odores são apresentados ao sistema de reconhecimento de padrões. Parte de fundamental importância no funcionamento dos narizes artificiais o sistema de reconhecimento de padrões é responsável por classificar os estímulos odorantes captados pelo sistema sensor. 
Os narizes artificiais têm sido utilizados com grande sucesso em diversas aplicações (Keller et al., 1995). Recentemente eles têm despertado especial interesse da indústria petrolífera, onde podem ser utilizados como dispositivos de segurança na detecção e reconhecimento de gases tóxicos. Em companhias petrolíferas, os narizes artificiais podem trabalhar na prevenção de vazamentos em dutos de transporte e em plataformas de extração e refino de derivados de petróleo. Posicionados em locais estratégicos, estes dispositivos podem detectar e identificar vazamentos químicos em estágios iniciais, antes que a concentração de gases tóxicos alcance níveis perceptíveis e perigosos para os seres humanos e natureza.

Tendo grande importância nos resultados produzidos por um nariz artificial, o sistema de reconhecimento de padrões tem recebido crescente atenção em seu desenvolvimento. Dentre as abordagens investigadas objetivando o reconhecimento de padrões, resultados promissores estão sendo encontrados no desenvolvimento de Sistemas Híbridos Inteligentes (Abraham e Nath, 2002; Mcgarry et al., 2002; Medsker, 1995). Os Sistemas Híbridos Inteligentes são modelos que resultam da combinação de duas ou mais técnicas computacionais distintas, visando unir vantagens para suprir deficiências individuais, na resolução de determinado problema.

Neste trabalho, procuramos investigar técnicas híbridas que possam contribuir para o aprimoramento do sistema de reconhecimento de padrões do protótipo de um nariz artificial (Santos, 2000), a ser utilizado na detecção de gases provenientes da indústria petrolífera. São abordadas redes neurais artificiais já consagradas na literatura no reconhecimento de odores, como redes neurais Multi-Layer Perceptron (MLP) (Rumelhart et al., 1986) e Time Delay Neural Network (TDNN) (Lang e Hilton, 1988); além de novas abordagens neurais híbridas como: a rede neuro-difusa Feature-weigthed Detector (FWD) (Li et al., 2002), capaz de efetuar a classificação de padrões, seleção dos atributos mais importantes e extração de regras explicativas difusas da rede; e a rede neural evolutiva Evolving Fuzzy Neural Network (EFuNN) (Kasabov, 1998), que opera de um modo on-line; aprende incrementalmente e manipula o conhecimento adquirido do ambiente a partir de regras difusas de classificação, realizando agregação, inserção e extração destas regras na arquitetura da rede. Para avaliar os classificadores, seus desempenhos serão comparados na classificação de duas bases de dados distintas, uma base de odores de vinhos e uma base de gases derivados da indústria petrolífera.

Este artigo está dividido em cinco seções. A seção 2 apresenta os classificadores investigados. Na seção 3 são descritos os experimentos realizados. Na seção 4 são apresentados os resultados obtidos com cada um dos classificadores na análise dos padrões de odores. As considerações finais são apresentadas na seção 5.

\section{SISTEMAS CONEXIONISTAS NO RE- CONHECIMENTO DE ODORES}

O reconhecimento de padrões em narizes artificiais têm sido proposto a partir de várias abordagens no decorrer da última década (Barbosa et al., 2002; Yamazaki et al., 2001; Santos, 2000; Natale et al., 1995; Gardner et al., 1994; Keller et al., 1994; Sundgren et al., 1991; Gardner et al., 1990). Na grande maioria das abordagens as redes neurais artificiais têm sido amplamente utilizadas no reconhecimento de odor, devido a sua capacidade de trabalhar com sinais não-lineares provenientes dos sensores, grande adaptabilidade, tolerância a erros, tolerância a ruído e paralelismo inerente, permitindo rapidez no uso após o treinamento (Gardner et al., 1990).

\subsection{Redes Multi-Layer Perceptron}

Dentre os vários modelos de redes neurais artificiais existentes, a rede MLP (Rumelhart et al., 1986) é a rede neural artificial mais difundida, parte por sua facilidade de implementação, parte por sua simplicidade. Características estas, que a tornaram uma das redes neurais artificiais mais utilizadas na classificação de padrões de odores em narizes artificiais (Yamazaki e Ludermir, 2001; Santos, 2000; Natale et al., 1995; Gardner et al., 1994; Keller et al., 1994; Sundgren et al., 1991; Gardner et al., 1990). Devido a sua ampla utilização no reconhecimento de odores, a rede MLP se torna necessária como parâmetro em qualquer tipo de comparação entre classificadores para narizes artificiais.

Nas simulações realizadas com a rede MLP foram utilizados dois métodos de pré-processamento: normalização dos dados, de modo a que ficassem compreendidos na faixa de valores entre -1 e 1; e o filtro Wavelet, para redução do ruído e compressão dos dados de odores.

Foi utilizada uma rede MLP, contendo apenas uma camada intermediária. Neste experimento foi utilizada a codificação 1-of-m, onde $m$ é o número de classes (Prechelt, 1994), desta forma a rede neural possui tantas saídas quanto forem o número de classes investigadas. As unidades de processamento das camadas, intermediária e de saída possuem função de ativação tangente hiperbólica (Prechelt, 1994). A rede contém todas as possíveis conexões de propagação direta (feedforward) entre camadas adjacentes, sem possuir conexões que ligam unidades de processamento de camadas nãoadjacentes. Em cada um dos experimentos, foram treinadas seis topologias de rede distintas (com 4, 8, 12, 16, 20 e 30 unidades de processamento na camada intermediária).

O algoritmo de treinamento utilizado é uma versão do método de Lenvenberg-Maquardt, descrita em (Lang e Hil- 
ton, 1988). Para cada topologia, foram realizadas 30 (trinta) treinamentos, com inicializações de valores diferentes e aleatórios para as conexões entre as camadas de processamento. A parada do treinamento ocorre se for satisfeito o critério $G L_{5}$ do Proben1 (Prechelt, 1994) por duas vezes (para evitar que o treinamento seja interrompido por oscilações iniciais no erro de validação). O critério $G L_{5}$ fornece uma idéia sobre a perda da generalização durante o treinamento da rede, sendo muito útil para evitar overfitting. Este parâmetro é definido como o aumento do erro de validação em relação ao erro de validação mínimo obtido. O treinamento também é interrompido se for satisfeito o critério de progresso de treinamento do Proben1 (Prechelt, 1994), com $P_{5}(t)<0.1$. Este parâmetro fornece uma idéia de quanto o erro médio de treinamento é maior que o mínimo erro de treinamento, durante uma seqüência de épocas consecutivas. $\mathrm{O}$ treinamento também é encerrado se a quantidade máxima de 5.000 iterações for alcançada.

\subsection{Rede Time Delay Neural Network}

A rede Time Delay Neural Network (Time Delay), foi proposta por (Waibel et al., 1989), com a finalidade de resolver o problema de reconhecimento de fonemas isolados utilizando um espectrograma. Em essência, uma TDNN é uma rede neural artificial com arquitetura feedforward, onde a resposta da rede no tempo $t$ é baseada nas entradas no tempo $(t-1),(t-2), \ldots,(t-n)$. Com isso, considera-se um histórico da seqüência temporal dos dados.

A abordagem utilizada neste trabalho foi proposta por (Yamazaki e Ludermir, 2001). Esta abordagem consiste de um sistema de reconhecimento de padrões capaz de analisar as características temporais dos sinais gerados pelos sensores do nariz artificial, levando em consideração a variação destes valores ao longo do intervalo de tempo em que foram feitas as aquisições dos dados.

Este trabalho tem particular interesse em redes TDNN, a qual, é a arquitetura conexionista que tem apresentado os resultados mais promissores na classificação de padrões de odores em narizes artificiais (Zanchettin e Ludermir, 2003c; Zanchettin e Ludermir, 2003b; Zanchettin e Ludermir, 2003a; Yamazaki e Ludermir, 2001; Yamazaki et al., 2001).

Como descrito em Yamazaki e Ludermir (2001), a arquitetura do sistema de reconhecimento de padrões necessita receber curvas completas geradas pelos sensores do nariz artificial durante a aquisição dos dados. Tais curvas precisam ser obtidas em intervalos ininterruptos de tempo, desta forma, apenas os valores obtidos em cada uma das repetições podem ser utilizados no conjunto de treinamento, validação e teste (os padrões não podem ser retirados da ordem de aquisição ou agrupados em conjuntos maiores).

Desta forma, uma das repetições da aquisição dos dados foi utilizada como conjunto de treinamento. Outras duas repetições distintas (com a mesma quantidade de dados) foram utilizadas como conjunto de validação e teste. Foram utilizadas as mesmas técnicas de pré-processamento adotadas nos experimentos com redes MLP.

Para o tratamento temporal dos padrões de odores, foi utilizada uma rede TDNN (Lang e Hilton, 1988), contendo apenas uma camada intermediária. A camada de entrada possui o dobro do número de sensores do nariz artificial, pelo fato da utilização do atraso no tempo (delay de t-1), para cada uma das curvas de entrada. Este atraso é formado pelo valor atual de resistência do sensor e por um valor temporalmente anterior a este, de cada um dos sensores. Esta quantidade de atrasos no tempo para cada uma das curvas de entrada foi escolhida por ser a menor configuração possível utilizando características temporais. Foi utilizada a mesma codificação, topologia, função de ativação e algoritmo de aprendizado adotados na rede MLP descrita na seção anterior. Da mesma forma, foram realizadas trinta (30) execuções com diferentes inicializações dos valores das conexões entre as camadas de processamento. Os critérios de parada do treinamento foram os mesmos adotados nos experimentos com a rede MLP.

É importante ressaltar que os erros analisados foram computados separadamente para cada odor, ou seja, a rede avaliou, para cada sensor, as curvas correspondentes a cada independentemente, e não uma única curva contendo os valores dos odores em sequiência. Isto foi adotado para que a ordem de apresentação dos odores à rede não influencie nos resultados obtidos.

\subsection{Rede Feature-weighted Detector}

A abordagem neuro-difusa Feature-weighted Detector ( $\mathrm{Li}$ et al., 2002), consiste de uma rede com conexões de memória para classificação de padrões e conexões de pesos para seleção de atributos. Esta abordagem possui a capacidade de selecionar as características importantes de um conjunto de dados, enquanto mantém a taxa máxima de reconhecimento de padrões. Além da capacidade de selecionar atributos e classificar padrões, o método possibilita a extração do conhecimento adquirido pela rede no processo de aprendizagem na forma de um conjunto de regras interpretáveis do tipo $\mathrm{Se}$ Então.

Este modelo oferece especial interesse no tratamento dos padrões de odores por se propor a resolver dois dos principais problemas do reconhecimento de padrões: a seleção de atributos e a classificação dos padrões, além da capacidade de 
extrair regras difusas da estrutura da rede. Estas regras podem ser utilizadas na explicação do conhecimento adquirido pela rede na fase de aprendizagem, resolvendo assim uma conhecida deficiência das redes neurais artificiais.

A partir de experimentos prévios, realizados com a rede FWD, verificou-se que a ordem com que os padrões de odores são apresentados no treinamento, possui influência sobre seu desempenho na classificação dos odores. Assim, nos experimentos, os exemplos de cada um dos odores foram apresentados de forma ordenada no treinamento da rede (primeiramente foram apresentados todos os exemplos de um tipo de odor, posteriormente de outro tipo, etc.). Para o teste da rede neuro-difusa os padrões de odores foram apresentados de forma aleatória.

A fim de considerar esta necessidade de ordenação e verificar o desempenho da rede FWD com várias partições de dados, os mesmos conjuntos empregados nos experimentos com a rede TDNN foram utilizados. A rede FWD não utiliza conjunto de validação em seu treinamento. É importante salientar que os conjuntos de treinamento e teste de cada uma das partições são formados por diferentes repetições de aquisições de dados e que as características das respostas dos sensores podem se modificar de uma aquisição para outra (Santos, 2000).

O conjunto de padrões foi normalizado de modo a ficar compreendido na faixa de valores entre 0 e 1 . O préprocessamento a partir do filtro Wavelet não foi utilizado nos experimentos com a rede FWD, pois em análises preliminares não melhoraram os resultados apresentados pelo modelo. Por questão de objetividade seus resultados foram suprimidos das simulações realizadas com a rede FWD.

Foram realizadas 30 (trinta) execuções, para cada uma das partições. São utilizadas inicializações baseadas nos centros dos dados para as conexões de memória e valores fixos em 1 para as conexões de pesos. Os critérios de parada do treinamento utilizados foram o erro mínimo de treinamento $\epsilon=0$ e o número máximo de 5.000 iterações.

\subsection{Rede Evolving Fuzzy Neural Network}

A abordagem evolutiva neuro-difusa EFuNN (Kasabov, 1998), é uma estrutura conexionista que possui características como: aprendizado rápido (possivelmente em modo onepass learning), incremental e on-line; sua estrutura conexionista pode ser interpretada por regras difusas; utiliza regras de classificação para o crescimento, poda e agregação de sua topologia; e suas unidades de processamento e as conexões entre estas unidades são criadas, eliminadas e agregadas dinamicamente durante a operação da rede.
Toda a estrutura da rede EFuNN é construída sobre um conjunto de regras difusas, onde é possível modificar dinamicamente a topologia da rede. Assim, o modelo pode se adaptar a novos dados de treinamento (inclusive inserindo novas classes) de uma maneira on-line e aprender incrementalmente a partir de características locais dos exemplos de treinamento. Todo o conhecimento adquirido pela rede pode ser expresso na forma de regras de classificação difusas do tipo Se-Então.

A arquitetura EFuNN é denominada evolutiva porque sua topologia cresce com a apresentação de novos dados na fase de aprendizado e regularmente diminui através de poda (prunning), ou através da agregação de unidades de processamento de regras.

O interesse na aplicação da rede EFuNN no tratamento dos padrões de odores surgiu a partir de três de suas funcionalidades: (1) possibilidade de extração do conhecimento da rede a partir de regras de classificação; (2) aprendizado incremental, o qual possibilita que novos odores sejam aprendidos durante a operação do dispositivo; e (3) treinamento on-line, que possibilita a apresentação de novos padrões e classes a rede, sem a necessidade de re-apresentar toda a base de dados previamente treinada. Em um dispositivo onde se prima pela eficiência, rapidez, plasticidade e tamanho estas características são muito apreciadas.

Pelo fato da rede EFuNN não utilizar conjunto de validação em seu treinamento, nos experimentos realizados a base de dados utilizou somente dois conjuntos de dados: conjunto de treinamento, correspondendo a $50 \%$ da quantidade total de padrões; e conjunto de teste, correspondendo a $25 \%$ dos dados. Foram utilizados os mesmos dados empregados no treinamento da rede MLP.

A rede EFuNN não necessita de pré-processamento, desta forma, os dados são apresentados à camada de entrada sem processamento algum. Nesta camada os exemplos são transformados em padrões difusos, representados pela segunda camada de processamento da rede, no espaço de representação do problema. Desta forma, os padrões de odores foram apresentados à rede com a mesma amplitude de valores captada pelo sistema sensor do nariz artificial. Cada unidade de processamento de entrada e saída contém três funções de pertinência (Baixo, Médio e Alto), utilizadas na representação difusa dos dados.

A inexistência de aleatoriedade no treinamento da rede EFuNN faz com que uma execução da rede seja suficiente para fornecer uma idéia sobre a generalização do modelo. Ou seja, como o aprendizado é realizado a partir dos exemplos apresentados ao modelo, os mesmos dados irão convergir sempre para um mesmo ponto no espaço de busca. Assim, 
para realizar a comparação da rede EFuNN com as demais abordagens investigadas neste trabalho foram criadas 30 partições, de forma a se obter um erro médio de classificação para a base de odores utilizada.

O modo de treinamento utilizado foi one pass training, os exemplos são apresentados à rede somente uma vez para cada uma das modalidades e partições testadas. Foram realizadas quatro modalidades de experimentos: (1) no primeiro experimento a rede foi inicializada sem nenhuma regra e em seu treinamento não foram realizadas operações de poda ( $p r u$ ning) ou agregação de nodos de regras; (2) no segundo experimento, a rede também foi inicializada sem nenhuma regra, mas durante seu treinamento foi utilizada a técnica de poda dos nodos de regras que estivessem fora do limiar de ativação pré-estabelecido; (3) no terceiro experimento, a rede foi inicializada com algumas regras, remanescentes de treinamentos prévios e novos dados foram apresentados à rede de maneira on-line. Durante este treinamento foram utilizados ambos, poda e agregação; e (4) no quarto experimento, a partir de uma arquitetura previamente treinada com alguns odores, foram apresentados exemplos novas classes de odores, forçando a rede a se adaptar a estes novos exemplos e englobar automaticamente esta nova classe em sua topologia.

\section{EXPERIMENTOS}

O processo de aquisição dos estímulos odorantes pelo nariz artificial é realizado no sistema sensor, o qual é composto por uma matriz de sensores que captam informações do ambiente e as convertem em medidas quantitativas de interesse.

O sistema sensor pode ser formado por um conjunto de sensores distintos, em que cada elemento mede uma propriedade diferente do composto odorante, ou por um único dispositivo que produz um conjunto de medições para cada composto odorante. O sistema sensor pode também ser formado por uma combinação das estruturas descritas anteriormente (Yamazaki e Ludermir, 2001; Keller et al., 1995; Keller et al., 1994). Vários tipos de sensores e dopantes característicos podem ser utilizados na construção da matriz de sensores, dependendo da resposta desejada ou do odor analisado. Desta forma, todo composto odorante apresentado ao sistema sensor produz nos sensores alterações físicas ou químicas que o caracterizam.

Neste trabalho utilizamos duas bases de dados distintas, obtida a partir da exposição do protótipo de um nariz artificial (Santos, 2000) frente a dois compostos distintos. A matriz de sensores é composta pelo arranjo de sensores baseados em filmes de polipirrol ${ }^{1}$. Os sensores foram preparados com diferentes dopantes, por polimerização in situ, em substra-

\footnotetext{
${ }^{1}$ Polímero Condutor, material orgânico, do tipo "plástico", geralmente derivado do petróleo, que conduz eletricidade.
}

tos de ITO (substrato vítreo condutor). Algumas vantagens na utilização deste tipo de sensor são: (1) rápida cinética de absorção em temperatura ambiente; (2) pequeno consumo de energia (da ordem de microwatts), pois não é necessário um elemento aquecedor; (3) resistência ao envenenamento por compostos que normalmente tornariam inativos os sensores inorgânicos de semicondutores, como os compostos que contém enxofre; e (4) a possibilidade de o polímero ser construído com especificidade para determinados compostos químicos (Persaud e Travers, 1997).

A aquisição dos dados foi realizada de forma automática pelo nariz artificial, estando os sensores expostos aos odorantes em uma câmara especial de testes. A concentração de cada um dos odores foi constante em cada uma das aquisições dos dados e entre as diferentes aquisições. Maiores detalhes sobre a construção do protótipo podem ser encontrados em (Santos, 2000).

A primeira base trata da classificação de odores provenientes de três safras distintas (anos 1995, 1996 e 1997) de um mesmo vinho tinto comercial (Almadém, Brasil) produzido com uvas do tipo merlot. Foram realizadas três repetições de aquisição de dados. Em cada uma destas repetições, para cada uma das três safras de vinho, o valor da resistência de cada um dos seis sensores utilizados foi registrado de meio em meio segundo, sendo que o processo estendeu-se por cinco minutos. Dessa forma, cada sensor obteve 600 valores registrados para cada safra de vinho. O conjunto formado pelos seis valores dos seis sensores no mesmo instante de tempo foi considerado como um padrão da base de dados. Assim, cada repetição contém 1.800 padrões (600 de cada safra). Como existem três repetições, tem-se um total de 5.400 padrões na base de dados de vinhos.

A segunda base de dados provém da exposição do nariz artificial frente a compostos fornecidos pela pela companhia petrolífera Petrobrás ${ }^{2}$. Os testes foram realizados frente aos gases derivados do petróleo: Metano, Etano, Propano e Butano. Também foi verificada a sensibilidade frente ao gás Monóxido de Carbono. Os dados foram obtidos em nove repetições de testes, para os cinco gases analisados. Em todas as repetições, o valor de resistência de cada um dos oito sensores utilizados foi registrado em intervalos de 20 segundos. Como o processo de aquisição dos padrões de cada arranjo estendeu-se por 40 minutos, cada sensor obteve o registro de 120 valores, para cada um dos cinco gases apresentados ao nariz artificial. Desta forma, cada repetição contém 600 padrões, formados por 960 valores de cada gás. A partir das nove repetições, obteve-se um total de 5.400 padrões que formam a base de dados de gases. A tabela 1 apresenta um exemplo de aquisição dos dados para o gás Butano. Os sensores utilizados são denominados pelo nome do do-

\footnotetext{
${ }^{2}$ Petróleo Brasileiro S. A., Brasil.
} 
pante utilizado em sua constituição, DBS - Dodecilbenzonato de Sódio, OSA - Octil-sulfato de Sódio, NBS - Ácido Nitrobenzeno Sulfônico, ASA - Antraquinona Sulfonato de Sódio, PTSA - Ácido P-toluenosulfônico, CAS - Ácido Canforsulfônico, PER - Perclorato de Lítio, OSA2 - Octil-sulfonato de Sódio.

Tabela 1: Exemplo de uma das repetições de aquisição dos dados, para o gás Butano.

\begin{tabular}{ccccccccc}
\hline $\begin{array}{c}\text { Tempo } \\
\text { (s) }\end{array}$ & \multicolumn{8}{c}{$\begin{array}{c}\text { Sensores } \\
\text { (valores de resistência) }\end{array}$} \\
\hline \multicolumn{1}{c}{ DBS } & OSA & NBS & ASA & PTSA & CAS & PER & OSA2 \\
\hline 20 & 684 & 360 & 6140 & 141,6 & 269,2 & 3222 & 3238 & 498 \\
40 & 686 & 360 & 6140 & 141,6 & 269,4 & 3228 & 3236 & 498 \\
60 & 686 & 360 & 6140 & 141,6 & 269,4 & 3222 & 3234 & 498 \\
80 & 688 & 360 & 6140 & 141,6 & 269,4 & 3228 & 3236 & 500 \\
$\ldots$ & $\ldots$ & $\ldots$ & $\ldots$ & $\ldots$ & $\ldots$ & $\ldots$ & $\ldots$ & \\
2.400 & 920 & 360 & 6180 & 141,8 & 270,8 & 3250 & 3182 & 500 \\
\hline
\end{tabular}

Para auxiliar na análise dos experimentos que serão realizados neste estudo a Análise de Componentes Principais (PCA) (Jackson, 1991) foi empregada na visualização dos padrões nas duas bases de dados. Neste tipo de análise é essencial a escolha do número de componentes principais (CPs) utilizados na descrição do sistema, de modo a reduzir a dimensão das amostras estudadas. Serão consideradas as três primeiras CPs, pois juntas correspondem a mais de $97.5 \%$ e $99.5 \%$ da variância total dos padrões analisados nos dados das bases de vinhos e gases, respectivamente.

A projeção dos escores da base vinhos, nas três primeiras componentes principais, pode ser vista na figura 1 . Pode ser verificado que as amostras das Safras 95 e 97 estão mais agrupadas, enquanto que as amostras da Safra 96 está bem separada das demais.

A projeção dos escores da base gases é apresentada na figura 2. A partir desta figura pode-se observar que a distinção entre a maioria dos gases é bem definida, apenas os gases Metano e Etano mantêm-se agrupados em um plano de difícil separação.

A PCA pode também ser utilizada para verificar a importância de cada uma das variáveis do conjunto original de dados. Ou seja, as variáveis originais ou os sensores em nosso caso, com maior peso na combinação linear dos primeiros componentes principais são os mais importantes do ponto de vista estatístico.

A partir da análise dos pesos de cada uma das variáveis, foi possível verificar quais sensores mais contribuem para a separação dos odores em cada uma das componentes principais. Verificou-se que na separação dos vinhos três sensores

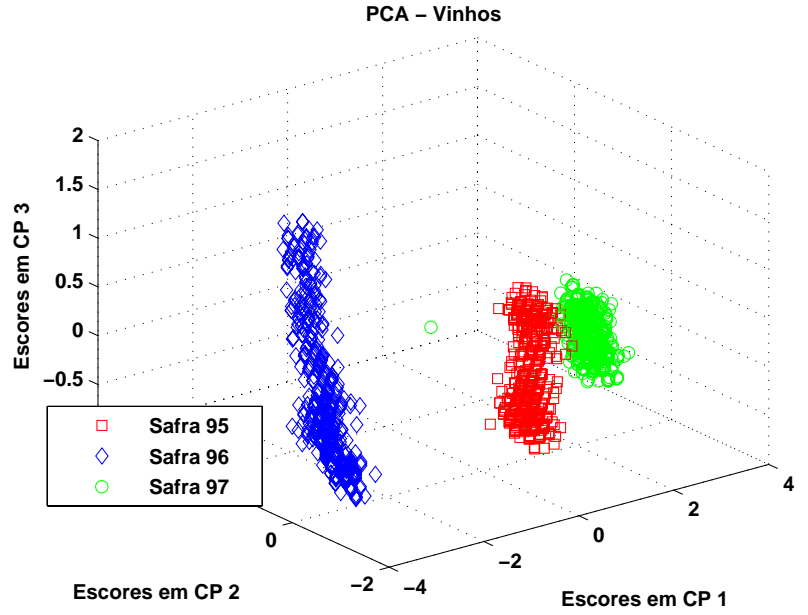

Figura 1: Projeção dos escores nas três primeiras CPs de 1800 padrões da base de vinhos.

possuem maior relevância, são eles: Dodecilbenzonato de Sódio - DBS, Antraquinona Sulfonato de Sódio - ASA, Octilsulfonato de Sódio - OSA. Na base de gases verificou-se que cinco sensores são os mais relevantes: Dodecilbenzoato de Sódio - DBS, Octil-sulfonato de Sódio - OSA, Antraquinona Sulfonato de Sódio - ASA, Perclorato de Lítio - PER e Octilsulfonato de Sódio - OSA2, possuem maior relevância para a separação dos padrões nas componentes principais analisadas.

\subsection{Pré-Processamento dos Dados}

Nos experimentos realizados, foram criadas 3 partições de dados, baseadas na base de dados original. Cada uma das partições possui suas instâncias dispostas em diferentes ordens de forma aleatória. A partir destas partições, a base de padrões de odores foi dividida em três conjuntos de dados, os quais correspondem aos conjuntos de treinamento, validação e teste utilizados nos experimentos com os classificadores. A proporção de padrões contidos em cada um dos conjuntos foi a seguinte: $50 \%$ dos padrões de odores foram dispostos no conjunto de treinamento; $25 \%$ dos padrões correspondem ao conjunto de validação; e os $25 \%$ restantes foram utilizados no conjunto de teste.

A utilização de partições de dados é necessária para eliminar qualquer viés existente no treinamento das redes neurais artificiais. Este tipo de divisão nos padrões de treinamento é sugerida pelo relatório Probenl (Prechelt, 1994), um conhecido Benchmark para o treinamento de redes neurais artificiais. 


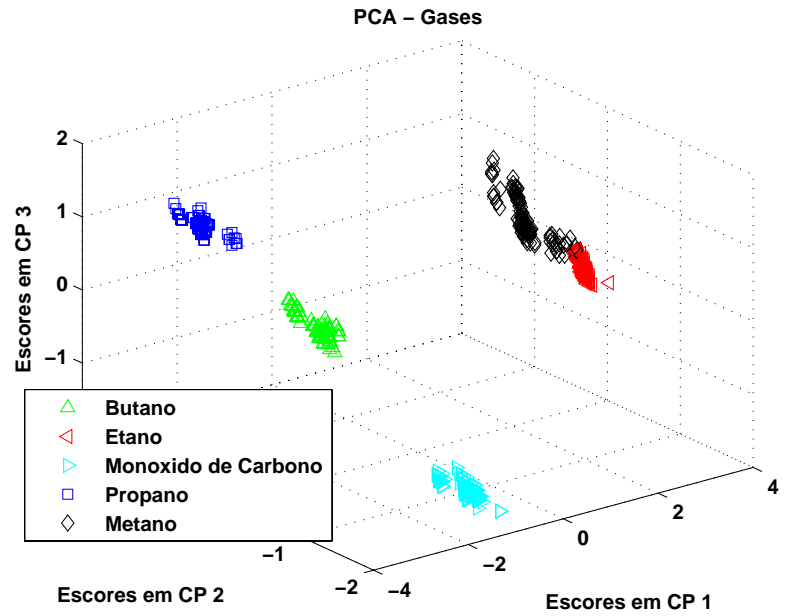

Figura 2: Projeção dos escores nas três primeiras CPs de 600 padrões da base de gases.

\subsubsection{Normalização dos Dados}

Para uma maior efetividade e rapidez no treinamento de redes neurais artificiais, é aconselhável a transformação de todos os atributos de valores reais para um mesmo intervalo. Dados com atributos em escalas diferentes podem confundir o aprendizado da rede, fazendo que esta tenha maior consideração por valores com grandes magnitudes. As técnicas de normalização evitam este tipo de problema, transpondo os dados em intervalos bem definidos.

Podem ser utilizadas normalizações Euclidianas, logarítmicas ou funções trigonométricas, tais como: tangente hiperbólica, seno, coseno, etc. Nos experimentos, os padrões de odores foram normalizados separadamente para cada sensor do nariz artificial. Duas formas de normalização foram utilizadas: (1) normalização nos dados para que os valores ficassem dispostos entre -1 e 1; e (2) normalização para que ficassem dispostos entre 0 e 1 .

\subsubsection{Filtro Wavelet}

Ao considerarmos que os sinais odorantes captados pelo nariz artificial são sinais, adquiridos por um sensor em um determinado intervalo de tempo; uma das ferramentas mais indicadas para o tratamento destes padrões são técnicas utilizadas no processamento de sinais digitais. A partir deste pressuposto, a Transformada Wavelet (Daubechies, 1990) foi utilizada como método de pré-processamento dos sinais.

A Transformada Wavelets é uma ferramenta matemática que possui vantagens perante técnicas clássicas de processamento de sinais, como a Análise de Fourier (Brigham, 1988): suas funções bases (wavelets) não pertencem a um espaço finito de soluções, isto é, existem teoricamente infinitas possibilidades de se projetar wavelets com propriedades especiais, voltadas para aplicações específicas. A Análise Wavelet é uma transformação integral onde os núcleos de integração usados na transformação do sinal são denominados de wavelets. Estas wavelets são essencialmente utilizadas de duas maneiras no estudo de processos ou sinais: (1) como um núcleo de integração para analisar a extração de informações sobre um processo; e (2) como uma base de representação ou caracterização do mesmo.

A figura 3 ilustra o processo onde a resolução de tempodomínio de um sinal $x(k), k=1, \ldots, N$ é alterado pela operações de filtragem passa/faixa e a escala é alterada por operações de downsampling/upsampling. Os parâmetros da Transformada Wavelet são o tipo do filtro Wavelet utilizado e o número de níveis de decomposição $(l=1, \ldots, L)$.

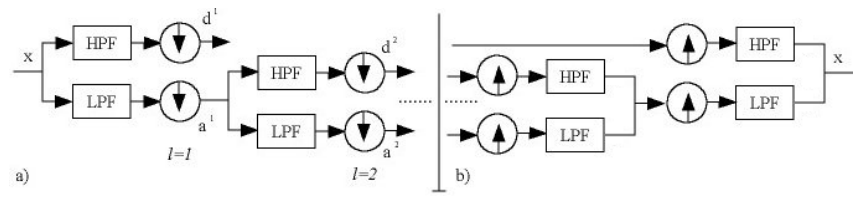

Figura 3: Transformada Wavelet: a) Forward e b) Inversa.

Em níveis baixos da escala de decomposição, a definição de tempo é negligenciada em favor de uma melhor definição da freqüência. Deste modo eventos de baixa freqüência podem ser melhor analisados com respeito a seu índice de freqüência mas não com respeito a sua posição no tempo. Em níveis elevados da escala uma melhor definição do tempo é obtida, a posição de eventos de alta frequiência é bem localizada no tempo, mas seu índice de freqüência real não é exato.

A Transformada Wavelet pode ser representada por:

$$
\begin{gathered}
d_{0,0}=\langle g(t), \phi(t)\rangle \\
d_{j, k}=\left\langle g(t), \psi_{j, k}(t)\right\rangle, j=1, \ldots, N, k=1, \ldots, 2^{j-1}
\end{gathered}
$$

onde $d_{j, k}$ são os coeficientes wavelet, $g(t)$ é o sinal a ser transformado, $\phi(t)$ é a escala da função, $\psi_{j, k}(t)$ é a função wavelet mãe $\psi(t)$ dada por:

$$
\psi_{j, k}(t)=2^{j / 2} \psi\left(2^{j} t-k\right)
$$

e $N$ é o número de escalas sobre o qual a Transformada Wavelet é gerada. Seguindo esta notação, a Transformada Wavelet Inversa pode ser dada por:

$$
g(t)=d_{0,0} \phi(t)+\sum_{j, k} d_{j, k} \psi_{j, k}(t)
$$


O filtro Wavelet utilizado neste trabalho foi construído com a Transformada Wavelets da família Daubechies de ordem 3 (Daubechies, 1988), com threshold de filtragem fixo $(t h r=$ 35). Este filtro é utilizado para redução do ruído inerente ao sinal de odor e como método de compressão deste sinal. Como o método de aquisição dos dados no nariz artificial induz a ruídos de baixa frequiência, os sinais livres de ruído foram encontrados na componente de mais baixa freqüência de uma árvore de 3 níveis de decomposição.
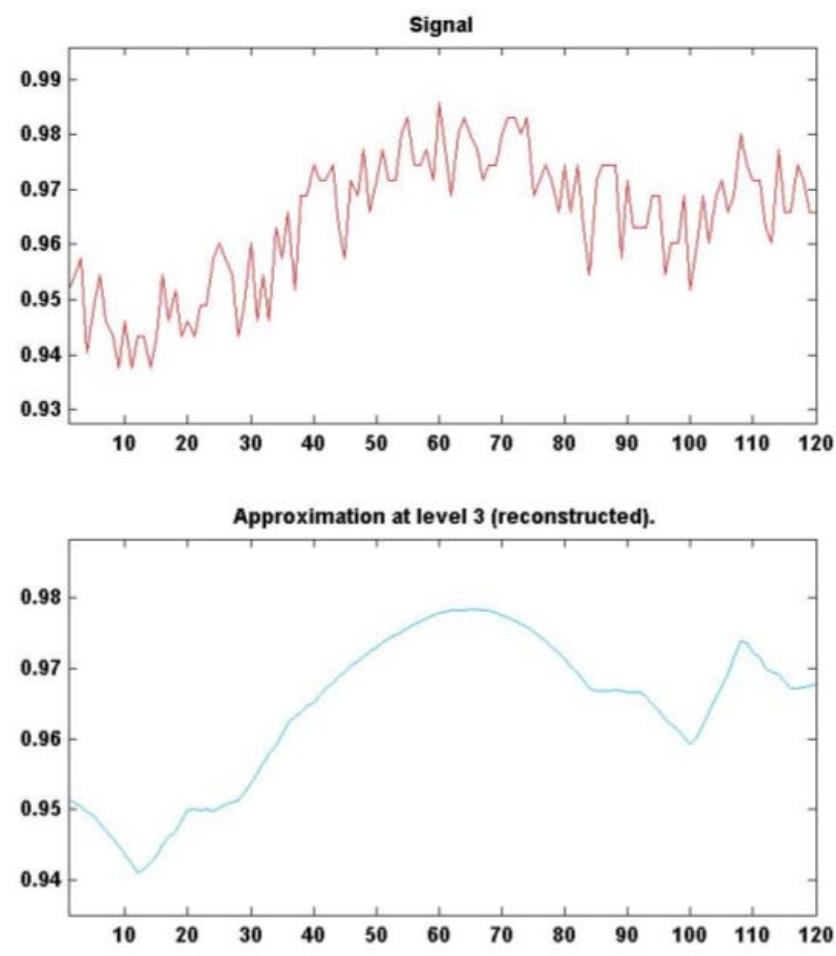

Figura 4: Decomposição utilizada para filtrar o ruído e comprimir o sinal de odor.

Na figura 4 pode ser verificada a decomposição do sinal analisado. A partir desta decomposição o sinal de odor original foi reconstruído a partir do terceiro nível de decomposição sem os componentes ruidosos que poderiam prejudicar o desempenho dos classificadores. A taxa de compressão obtida foi de $85.71 \%$. O filtro foi implementado utilizando o Toolbox Wavelets (Misiti et al., 1996), do software de simulação Matlab 6.5.1 ${ }^{3}$.

\subsection{Método de Comparação entre as abordagens}

$\mathrm{Na}$ maioria das áreas de pesquisa há uma busca contínua por novos métodos que melhorem em algum sentido o desem-

${ }^{3}$ The MathWorks, Inc. penho das abordagens existentes. Na agricultura, por exemplo, buscam-se variedades mais adequadas e mais produtivas de cereais. Na computação, buscam-se técnicas que melhorem a qualidade e o desempenho dos softwares existentes. Nestas situações é preciso comparar as técnicas usuais com os métodos alternativos propostos. Esta comparação é uma questão importante que surge freqüentemente no trabalho de pesquisa. Para se chegar a qualquer conclusão é necessário coletar informações e fazer inferências a partir de evidências experimentais ou observacionais.

A partir das observações realizadas, para uma resposta coerente, é necessário generalizar os resultados obtidos no grupo estudado para a população de interesse. Esta tarefa exige cuidados especiais no planejamento dos experimentos, sendo que fatores que afetem a resposta avaliada devem ser controlados e considerados. O procedimento utilizado para determinar se o resultado de uma análise aponta para sim ou para não é chamado Teste de Hipótese (Duda et al., 2001). Este procedimento é amplamente utilizado nas áreas de pesquisa onde as variáveis envolvidas estão sujeitas à grande variabilidade. O teste de hipóteses utilizado na comparação dos classificadores investigados neste trabalho é o teste de Wilcoxon (Johson e Wichern, 1999).

O teste de Wilcoxon, também conhecido por Teste dos Sinais por Postos, é um teste de hipóteses não-paramétrico para dados pareados. O teste de hipóteses será utilizado para comparar os resultados obtidos por cada um dos classificadores. A partir destes testes será possível afirmar qual dos classificadores avaliados possui o melhor desempenho na classificação dos compostos odorantes. Os resultados foram considerados estatisticamente significativos quando $\mathrm{P}<0,05$.

\section{RESULTADOS}

\subsection{Redes Multi-Layer Perceptron}

Foram realizados dois experimentos distintos: (1) um experimento onde o método de pré-processamento utilizado é somente a normalização dos padrões captados pelo sistema sensor do nariz artificial, de modo que estes valores fiquem compreendidos no intervalo [-1, 1]; e (2) um experimento que utiliza um filtro Wavelet para redução do ruído presente no sinal captado pelo sistema sensor do nariz artificial e como um mecanismo de compressão para os dados de odor.

Na tabela 2 são apresentados os resultados do primeiro experimento, sem o uso do filtro Wavelet. Para cada um dos experimentos apresenta-se uma tabela contendo o nome da base de dados, a melhor topologia encontrada, a média dos resultados obtidos nas trinta (30) execuções realizadas, com as três partições (Sum Squared Error - SSE e Erro de Classificação), bem como os valores de média e desvio-padrão. 
Tabela 2: MLP - Experimento 1.

\begin{tabular}{lccccccc}
\hline & & \multicolumn{2}{c}{$\begin{array}{c}\text { SSE de } \\
\text { Base }\end{array}$} & Nodos & \multicolumn{2}{c}{$\begin{array}{c}\text { SSE de } \\
\text { Treinamento }\end{array}$} & \multicolumn{2}{c}{$\begin{array}{c}\text { Erro de } \\
\text { Validação }\end{array}$} & \multicolumn{2}{c}{\begin{tabular}{c} 
Classificação \\
\hline
\end{tabular}} & \multicolumn{3}{c}{ Média } & Desvio & Média & Desvio & Média & Desvio \\
\hline Vinhos & 8 & 598,63 & 598,63 & 295,28 & 295,28 & $\mathbf{0 , 1 0 9 6}$ & 0,1096 \\
Gases & 8 & 1849,76 & 2681,81 & 967,09 & 1379,38 & $\mathbf{0 , 1 4 6 1}$ & 0,1913 \\
\hline
\end{tabular}

Em ambas as bases o menor erro de validação e classificação foi obtido pela topologia com 8 nodos na camada escondida. Correspondendo a um erro de classificação médio de $10.96 \%$ para a base de vinhos e $14.61 \%$ para a base de gases (tabela 2). Foi verificado também que a partir da topologia com 8 nodos na camada intermediária, o erro obtido pela rede neural aumenta com a adição de novas unidades de processamento nesta camada. Apesar deste indício, foram realizados experimentos com topologias onde o número de unidades de processamento na camada intermediária ultrapassava 30 nodos (com 50, 70, 100, 150 e 300 unidades de processamento na camada intermediária). Porém, nestes experimentos o erro de classificação manteve a mesma tendência de alta, proporcional ao número de unidades de processamento adicionadas.

Na tabela 3 são apresentados os resultados do treinamento realizado com as redes MLP utilizando o filtro Wavelet.

Tabela 3: MLP com filtro Wavelet - Experimento 2.

\begin{tabular}{lccccccc}
\hline & & \multicolumn{2}{c}{$\begin{array}{c}\text { SSE de } \\
\text { Base }\end{array}$} & Nodos & \multicolumn{2}{c}{$\begin{array}{c}\text { SSE de } \\
\text { Treinamento }\end{array}$} & \multicolumn{2}{c}{$\begin{array}{c}\text { Erro de } \\
\text { Validação }\end{array}$} & \multicolumn{2}{c}{\begin{tabular}{c} 
Classificação \\
\hline
\end{tabular}} & Média & Desvio & Média & Desvio & Média & Desvio \\
\hline Vinhos & 12 & 689,83 & 1526,04 & 344,69 & 762,52 & $\mathbf{0 , 0 9 4 7}$ & 0,2120 \\
Gases & 8 & 1425,66 & 2373,96 & 737,11 & 1209,03 & $\mathbf{0 , 1 1 5 0}$ & 0,1734 \\
\hline
\end{tabular}

A partir dos resultados apresentados na tabela 3 se percebe que a utilização do filtro Wavelet, melhora consideravelmente o desempenho do método de classificação. Os menores erros de validação e classificação foram obtidos pelas topologias com 12 e 8 nodos na camada intermediária, um erro médio de $9,47 \%$ e $11.50 \%$ sobre o conjunto de teste, para as bases de vinhos e gases, respectivamente.

Se compararmos os melhores resultados obtidos pelos dois experimentos (resultados obtidos pelo classificador sem e com a utilização do filtro Wavelet) podemos verificar que a melhora foi substancial.

A fim de verificarmos a relevância estatística dos resultados obtidos, foi realizado o teste de hipóteses nestes experimentos. O teste é realizado a partir dos melhores resultados médios, obtidos por cada um dos experimentos (com e sem pré- processamento com filtro Wavelet). Neste teste, verificamos que apesar da menor média de classificação do segundo experimento, com nível de significância de 5\%, estatisticamente a performance média das redes MLP fazendo uso do filtro Wavelet é tão boa quanto à das redes que não usam este tipo de pré-processamento.

\subsection{Rede Time Delay Neural Network}

Os mesmos experimentos realizados com a rede MLP foram repetidos coma rede TDNN. Na tabela 4 são apresentados os resultados médios do treinamento, para as três partições testadas. Neste experimento somente foi utilizada a normalização, como método de pré-processamento.

Tabela 4: TDNN - Experimento 1.

\begin{tabular}{|c|c|c|c|c|c|c|c|}
\hline \multirow[t]{2}{*}{ Base } & \multirow[t]{2}{*}{ Nodos } & \multicolumn{2}{|c|}{$\begin{array}{c}\text { SSE de } \\
\text { Treinamento }\end{array}$} & \multicolumn{2}{|c|}{$\begin{array}{c}\text { SSE de } \\
\text { Validação }\end{array}$} & \multicolumn{2}{|c|}{$\begin{array}{c}\text { Erro de } \\
\text { Classificação }\end{array}$} \\
\hline & & Média & Desvio & Média & Desvio & Média & Desvio \\
\hline Vinhos & 8 & 0,09 & 0,13 & 22,40 & 13,88 & 0,0050 & 0,0227 \\
\hline Gases & 8 & 2,77 & 2,94 & 72,27 & 30,87 & $\mathbf{0 , 1 2 1 7}$ & 0,0869 \\
\hline
\end{tabular}

Como pode ser observado, a abordagem temporal diminuiu consideravelmente a variação dos resultados, refletido no baixo desvio-padrão apresentado pelos experimentos. As topologias com menor erro médio de validação e teste foram as topologias com 8 unidades de processamento na camada intermediária, para ambas as bases de dados. Foi obtido um erro médio de classificação de $0.50 \%$ e $12.17 \%$ para as bases de vinhos e gases, respectivamente. É importante salientar que os conjuntos de treinamento, validação e teste são formados por diferentes repetições de aquisição de dados, e as características das respostas dos sensores se modificam de uma repetição para a outra (Santos, 2000). A partir desta afirmação, pode-se perceber que a rede conseguiu generalizar muito bem o conhecimento adquirido em seu treinamento. Outro fato a ser observado nesta análise, da mesma forma que o ocorrido com as redes MLP, o acréscimo no número de unidades de processamento na camada intermediária não melhora o desempenho da rede.

Na tabela 5 são apresentados os resultados médios do segundo treinamento realizado com as redes TDNN, utilizando o filtro Wavelet. Como ocorreu nos experimentos com a rede MLP, a utilização do filtro Wavelet melhorou os resultados apresentados pela rede neural artificial. No caso das redes TDNN esta melhora foi considerável. NA base de gases o melhor resultado dos experimentos sem o filtro Wavelet foi um erro médio de classificação de $12.17 \%$ (tabela 4). Nos experimentos utilizando este filtro o erro médio de classificação foi de $0.75 \%$, obtido pela topologia com 8 unidades de processamento na camada intermediária. Na base de vinhos 
o erro de classificação obtido com a utilização do filtro Wavelet $(0.70 \%$ de erro - tabela 4$)$ manteve-se estável em relação aos resultados obtidos sem a utilização deste filtro $(0.70 \%$ tabela 5).

Tabela 5: TDNN com filtro Wavelet - Experimento 2.

\begin{tabular}{|c|c|c|c|c|c|c|c|}
\hline \multirow[t]{2}{*}{ Base } & \multirow[t]{2}{*}{ Nodos } & \multicolumn{2}{|c|}{$\begin{array}{c}\text { SSE de } \\
\text { Treinamento }\end{array}$} & \multicolumn{2}{|c|}{$\begin{array}{c}\text { SSE de } \\
\text { Validação }\end{array}$} & \multicolumn{2}{|c|}{$\begin{array}{c}\text { Erro de } \\
\text { Classificação }\end{array}$} \\
\hline & & Média & Desvio & Média & Desvio & Média & Desvio \\
\hline & 8 & 0,12 & 0,3 & 24,23 & 14,81 & & 47 \\
\hline Gases & 8 & 1,25 & 1,97 & 26,65 & 22,01 & 0,0075 & 0,0371 \\
\hline
\end{tabular}

Realizamos o teste de hipótese para verificar se esta melhora estatisticamente se confirma. Neste teste verificamos que a performance média das redes TDNN fazendo uso da análise Wavelet é melhor que a das mesmas redes não utilizando este tipo de pré-processamento.

\subsection{Rede Feature-weighted Detector}

Nos experimentos realizados, para cada uma das partições, os aspectos observados nas 30 execuções, bem como os valores de média e desvio-padrão são apresentados na tabela 6 . Nesta tabela, $\sigma$ representa a nebulosidade no agrupamento, $\alpha$ representa a taxa de aprendizado temporal e $\beta$ a taxa de aprendizado. A letra $x$ representa os atributos dos padrões de odores (sensores) e $w$ a média das conexões de pesos.

Tabela 6: Resultados da rede FWD - Experimento 1.

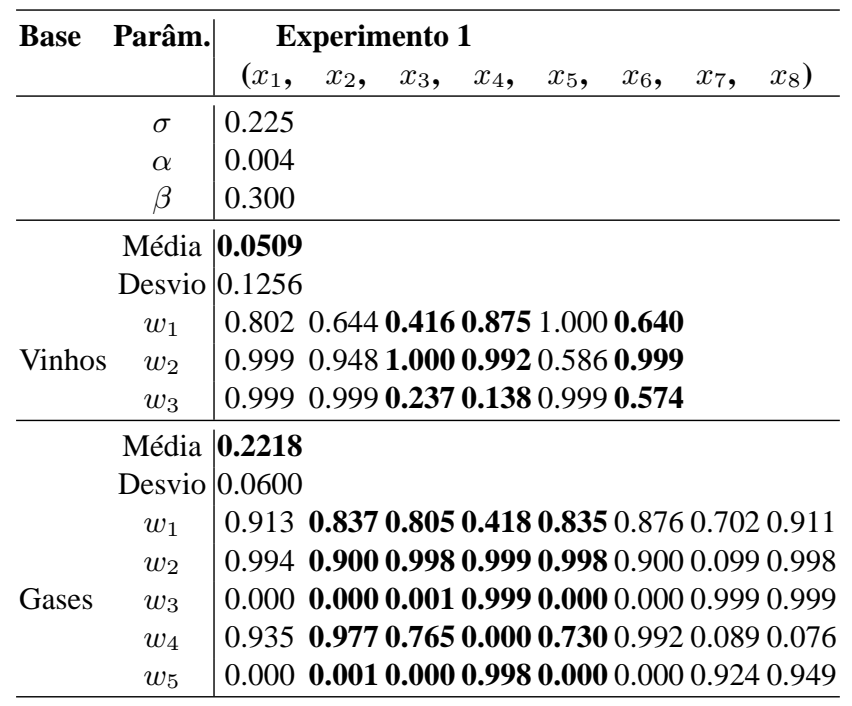

A rede FWD obteve dois resultados bem distintos na classificação dos dois tipos de odores. Na classificação da base de vinhos obteve uma boa generalização para o problema, um erro médio de classificação de $5.09 \%$ nas três partições de dados. Porém, esta afirmação não se verifica na classificação dos dados da base de gases, o erro médio de classificação obtido pela abordagem neuro-difusa foi de $22.18 \%$ (tabela 6). Um erro relativamente alto se considerarmos as abordagens previamente apresentadas. Em análises posteriores verificou-se que a rede apresenta dificuldades em separar os gases Etano e Metano, indicados na Análise de Componentes Principais como classes muito próximas. Isto pode caracterizar uma deficiência da rede FWD em tratar dados não-linearmente separáveis.

\subsubsection{Seleção de Atributos.}

No modelo FWD a seleção de atributos é realizada a partir dos valores das conexões de pesos obtidas durante a fase de treinamento da rede. Tais valores representam o grau de significância dos atributos para cada uma das classes.

Para verificar o desempenho do método de seleção de atributos, são realizados dois experimentos. No primeiro experimento, apresentado na tabela 6 , a rede foi treinada com os todos os atributos (representando os sensores) da base de dados de odores. Considerando estes resultados, pode-se observar para a base de vinhos: (1) os atributos $x_{3}, x_{4}$, e $x_{6}$ possuem pequena contribuição na formação dos agrupamentos das classes 1 e 3 ; e (2) a contribuição destes atributos para a classe 2 pode ser desempenhada pelos atributos $x_{1}$, e $x_{2}$. Na análise dos vetores de pesos da base de gases: (1) os atributos $x_{2}, x_{3}$ e $x_{5}$ possuem pequena contribuição na formação dos agrupamentos das classes 3 e 5 , e o atributo $x_{4}$ possui pequena participação nos agrupamentos das classes 1 e 4; (2) a contribuição dos atributos $x_{2}, x_{3}, x_{4}$ e $x_{5}$ para as classe 1, 3 e 4 pode ser desempenhada pelos demais atributos, que apresentaram grande contribuição na formação dos agrupamentos destas classes.

No segundo experimento, os atributos da base de vinhos $x_{2}$, $x_{3}$ e $x_{6}$ e da base de gases $x_{2}, x_{3}, x_{4}$ e $x_{5}$ foram excluídos e a rede foi novamente treinada somente com os atributos restantes ( $x_{1}, x_{2}$ e $x_{5}$ vinhos e $x_{1}, x_{6}, x_{7}$ e $x_{8}$ gases). No treinamento, foi utilizada a mesma configuração de parâmetros do primeiro experimento. Na base de vinhos a média do erro de classificação foi de $2.26 \%$, um erro menor que o obtido nos experimentos com 6 atributos. $\mathrm{Na}$ base de gases o erro se manteve, um erro médio de classificação de $20.16 \%$. Este resultado comprova a pequena relevância dos atributos removidos na formação dos agrupamentos. Os sensores indicados como de menor relevância foram: base de vinhos - Ácido Nitrobenzeno Sulfônico - NBS, Ácido P-toluenosulfônico - PTSA e Ácido Canforsulfônico - CAS; base de gases - Octil-sulfonato de Sódio - OSA, Ácido Nitrobenzeno Sulfônico - NBS, Antraquinona Sulfato de Sódio ASA e Ácido P-toluenosulfônico - PTSA. 


\subsubsection{Considerações.}

A fim de verificar a eficiência da seleção de atributos da rede FWD, foram novamente realizados dois experimentos com os demais modelos apresentados neste trabalho. Em um dos experimentos os classificadores foram treinados com todos os atributos da base de dados e no outro foram excluídos os atributos indicados pela rede FWD como de menor relevância para a formação dos agrupamentos de dados. A tabela 7 apresenta o teste estatístico realizado com os resultados médios destes experimentos. A hipótese nula $\left(H_{0}\right)$ é a de que os classificadores possuem o mesmo desempenho com e sem os atributos selecionados. A hipótese alternativa $\left(H_{1}\right)$ é a de que os classificadores possuem desempenhos diferentes.

Tabela 7: Análise dos dados.

\begin{tabular}{|c|c|c|c|c|c|c|}
\hline \multirow[t]{2}{*}{ Base } & \multicolumn{3}{|c|}{ Experimento 1 } & \multicolumn{2}{|c|}{ Experimento 2} & \multirow[b]{2}{*}{ Obs. } \\
\hline & Class. & Média & Desvio & Média & Desvio & \\
\hline \multirow{4}{*}{ Vinhos } & MLP & 0,1096 & 0,1096 & 0,0735 & 20 & 30 \\
\hline & TDNN & 0,0050 & 0,0227 & 0,0039 & & 30 \\
\hline & FWD & 0,0509 & 0,1256 & 0,0226 & & 30 \\
\hline & EFuNN & 0,0001 & 0,0003 & 0,0000 & 0,0000 & 30 \\
\hline \multirow{6}{*}{ Gases } & MLP & 0,1461 & 0,1913 & 0,1402 & & 30 \\
\hline & TDNN & 0,1217 & 0,0869 & 0,0982 & & 30 \\
\hline & FWD & 0,2218 & 0,0600 & 0,2016 & 0,0000 & 30 \\
\hline & EFuNN & 0,0088 & 0,0028 & 0,0333 & 0,0035 & 30 \\
\hline & \multicolumn{6}{|c|}{ Estatística do teste de Wilcoxon } \\
\hline & & & Valor $\mathbf{Z}$ & Valor $\mathbf{P}$ & Decisão & \\
\hline \multirow{4}{*}{ Vinhos } & MLP & & 702 & 0,470 & Não rejeitad & \\
\hline & TDNN & & $-0,405$ & 0,686 & Não $r$ & \\
\hline & FWD & & $-1,000$ & 0,317 & Não rejeitada & \\
\hline & EFuNN & & $-2,449$ & 0,014 & Rejeitada & \\
\hline \multirow{4}{*}{ Gases } & MLP & & 1000 & 0,276 & Não rejeit & \\
\hline & TDNN & & $-0,905$ & 0,365 & Não rejeitada & \\
\hline & FWD & & 3,992 & 0,001 & Rejeitada & \\
\hline & EFuNN & & 4,790 & 0,000 & Rejeitada & \\
\hline
\end{tabular}

A tabela 7 apresenta os resultados do teste de Wilcoxon para amostras emparelhadas, com nível de significância de 5\%. Os resultados comprovam a hipótese de que a performance dos modelos sem os referidos atributos é tão boa quanto o das redes treinadas com todos os atributos das bases de dados.

\subsection{Rede Evolving Fuzzy Neural Network}

Nesta seção serão apresentados os resultados obtidos nos 4 experimentos realizados com a rede EFuNN para cada uma das bases de dados. Na tabela 8 - simulação 1, são apresentados os resultados da primeira simulação, onde uma arquitetura EFuNN inicializada sem nenhuma regra é treinada com os padrões de odores das 30 partições formadas com cada uma das bases. Neste experimento, optou-se por não reali- zar poda ou agregação sobre os nodos de regras criados na camada de regras durante o treinamento.

Como pode ser visto, a rede EFuNN conseguiu uma boa generalização para o problema de classificação de odores. O erro de médio de classificação obtido pelas 30 partições foi de $0.15 \%$ e $0.88 \%$ para as bases de vinhos e gases, respectivamente. Uma taxa de erro extremamente baixa, considerando os resultados obtidos pelos demais classificadores.

No segundo experimento, apresentado na tabela 8 - simulação 2, a arquitetura EFuNN também foi inicializada sem nenhuma regra. Todos os nodos de regras foram criados dinamicamente durante o treinamento da rede. Porém, durante este treinamento é realizada a poda dos nodos de regras que estiverem fora do limiar de ativação estabelecido. Com a aplicação da técnica de poda, a quantidade de nodos de regras na rede diminuiu e por conseqüência a quantidade de regras extraídas da rede neuro-difusa também decresceu. No experimento anterior a quantidade média de regras foi de 345 e 134 regras, está média caiu para 17 e 79 regras, respectivamente para as bases de vinhos e gases.

A fim de verificar se o mecanismo de agregação poderia melhorar estes resultados (diminuir o erro de classificação em relação à segunda simulação e diminuir a quantidade de regras extraídas em relação à primeira simulação) e comprovar a capacidade da rede em aprender incrementalmente de maneira on-line. Neste experimento (tabela 8 - simulação 3) uma nova arquitetura EFuNN foi criada. Nesta arquitetura foram inseridas 10 regras, remanescentes de treinamentos prévios e novos padrões de odores foram apresentados a esta rede.

Este experimento nos permite verificar se o modelo realmente pode aprender ou se adaptar a novos padrões, sem perder ou "esquecer"o conhecimento previamente adquirido. Para isso, durante o treinamento da rede, foram utilizados os procedimentos de poda e agregação. Nesta simulação, na base de gases, houve uma melhora em relação aos resultados apresentados nos experimentos anteriores. O erro médio de classificação das 30 partições testadas foi de $2.82 \%$ e a quantidade média de regras extraídas foi de 102 regras. Para a base de vinhos os resultados se mantiveram, pois o número de regras na simulação 2 já era baixo (17 regras - tabela 8).

Vale ressaltar que este experimento possibilitou verificar além do treinamento on-line, a inserção de regras na topologia da rede. Pois, as regras utilizadas como conhecimento inicial na simulação, eram provenientes de um treinamento prévio e foram inseridas em uma arquitetura vazia de modo automático. Assim, o conhecimento pode ser inserido na rede EFuNN de duas formas distintas: a partir do treinamento da rede com novos exemplos ou com a inserção de regras previamente adquiridas. 
O quarto experimento da série de simulações realizadas com a rede EFuNN visa verificar as mais interessantes de suas funcionalidades para o reconhecimento de odores: o treinamento on-line e a inserção dinâmica de classes na arquitetura da rede.

A simulação foi realizada da seguinte forma: a partir de uma arquitetura previamente treinada com quatro gases e dois vinhos, escolhidos de forma aleatória, foram apresentados à rede exemplos de um quinto gás e uma terceira amostra de vinho. Nenhum dos exemplos do quinto gás ou da terceira safra de vinho foi apresentado à rede na fase anterior de treinamento. A inclusão desta nova classe de exemplos no treinamento da rede força o modelo a se adaptar a estes novos padrões e englobar o novo odor automaticamente em sua topologia. Desta forma, a rede foi primeiramente treinada com os gases: Butano, Etano, Monóxido de Carbono e Metano. $\mathrm{Na}$ segunda etapa do treinamento (inserção do quinto gás) o gás Propano foi apresentado à rede. Na base de vinhos primeiramente foram apresentadas amostras das Safras de 95 e 96 e posteriormente da Safra de 97.

Como pode ser verificado na tabela 8 - simulação 4 , o fato da terceira amostra de vinho e do quinto gás ter sido apresentado à rede posteriormente ao treinamento dos demais odores, não prejudicou em nada a generalização do modelo. Os testes na rede foram realizados com as três safras de vinho e os cinco gases, simultaneamente. O erro médio de classificação desta simulação foi de $0.32 \%$ e $0.66 \%$, porém a não utilização das técnicas de poda ou agregação pode ser percebida pela grande quantidade de regras extraídas da rede.

Tabela 8: Resultados dos experimentos realizados com a rede Evolving Fuzzy Neural Network

\begin{tabular}{|c|c|c|c|c|c|c|}
\hline Base & Sim. & $\begin{array}{c}30 \\
\text { Partições }\end{array}$ & $\begin{array}{c}\text { Erro } \\
\text { Treinamento }\end{array}$ & $\begin{array}{l}\text { Erro } \\
\text { Teste }\end{array}$ & $\begin{array}{c}\text { Erro } \\
\text { Class. }\end{array}$ & $\begin{array}{c}\text { Número } \\
\text { de Regras }\end{array}$ \\
\hline \multirow{8}{*}{ Vinhos } & 1 & Média & 0,0815 & 0,0805 & 0.00015 & 345 \\
\hline & & Desvio & 0,0060 & 0,0057 & 0,00030 & 12,81 \\
\hline & 2 & Média & 0,1602 & 0,1224 & 0,01365 & 17 \\
\hline & & Desvio & 0,0101 & 0,0832 & 0,03251 & 2,33 \\
\hline & 3 & Média & 0,1614 & 0,1299 & 0,01617 & 16 \\
\hline & & Desvio & 0,0111 & 0,0922 & 0,03808 & 2,32 \\
\hline & 4 & Média & 0,1205 & 0,1235 & 0,00324 & 312 \\
\hline & & Desvio & - & - & - & - \\
\hline \multirow{8}{*}{ Gases } & 1 & Média & 0,3651 & 0,2195 & 0,0088 & 134 \\
\hline & & Desvio & 0,0163 & 0,0462 & 0,0029 & 13,65 \\
\hline & 2 & Média & 0,5601 & 0,5082 & 0,0467 & 79 \\
\hline & & Desvio & 0,0064 & 0,0643 & 0,0122 & 6,35 \\
\hline & 3 & Média & 0,4275 & 0,3717 & $\mathbf{0 , 0 2 8 2}$ & 102 \\
\hline & & Desvio & 0,0139 & 0,0551 & 0,0081 & 8,09 \\
\hline & 4 & Média & 0,2469 & 0,1647 & 0,0066 & 228 \\
\hline & & Desvio & - & - & - & - \\
\hline
\end{tabular}

\section{CONSIDERAÇÕES FINAIS}

A construção de dispositivos que simulem o comportamento do sistema olfativo biológico é uma área em expansão. Muitas pesquisas estão sendo despendidas com a finalidade de simular a percepção humana. A maioria dos esforços visa refletir nos dispositivos eletrônicos a capacidade humana de perceber o mundo na forma de cinco sentidos ou até mesmo estender nossas potencialidades sensoriais.

O presente trabalho está contextualizado na construção de narizes artificiais, dispositivos eletrônicos cuja necessidade em diversas aplicações é indiscutível. O foco principal do artigo foi à construção de sistemas de reconhecimento de padrões para narizes artificiais, buscando técnicas que apresentem funcionalidades e resultados significativos na classificação de sinais de odorantes.

Foram abordados sistemas de reconhecimento de padrões como a rede Multi-Layer Perceptron (Rumelhart et al., 1986), a rede Time Delay Neural Network (Lang e Hilton, 1988) e abordagens híbridas, como as redes Featureweighted Detector (Li et al., 2002) e Evolving Fuzzy Neural Network (Kasabov, 1998). Além dos classificadores, foi avaliada a utilização da Transformada Wavelet (Daubechies, 1988) como filtro para redução do ruído inerente a captação do estímulo odorante e como método de compressão do sinal proveniente do sistema sensor.

Nos resultados obtidos com as arquiteturas conexionistas, a rede TDNN confirmou sua superioridade em relação às redes MLP. Quando aliada ao método de pré-processamento com o filtro Wavelet a rede TDNN obteve um erro médio de classificação de $0.70 \%$ e $0.75 \%$, enquanto a rede MLP obteve um erro médio de $9.47 \%$ e $11.5 \%$ sobre o conjunto de teste, nas bases de vinhos e gases respectivamente.

Dentre as arquiteturas híbridas verificadas, a rede FWD apresentou um desempenho de classificação baixo, em relação às demais abordagens investigadas na classificação da base de gases. A rede FWD apresentou um erro de classificação médio de $22.18 \%$. Na classificação da base de vinhos ela obteve um bom desempenho, um erro de classificação de 5.09\% sobre o conjunto de teste. Apesar do baixo desempenho sobre a base de gases, o método de seleção de atributos demonstrou excelentes resultados em ambas as bases. Estes resultados podem ser comparados aos obtidos com a técnica de Análise de Componentes Principais, na indicação dos atributos (ou sensores) mais relevantes na classificação dos odores. Com a utilização do método de seleção de atributos da rede FWD foi possível identificar os sensores do nariz artificial que apresentam maior relevância na classificação dos odores investigados. Também foi possível diminuir o tamanho da base de dados e consequientemente a complexidade da tarefa de classificação dos odores, visto que esta é diretamente pro- 
porcional ao número de sensores utilizados na detecção dos odores.

Apesar do promisor resultado na indicação dos atributos mais relevantes para classificação dos odores, o método de seleção de atributos da rede FWD apresenta uma deficiência, a grande subjetividade para a escolha dos atributos menos relevantes. A rede FWD também permite a extração de regras difusa de sua arquitetura, porém somente pode ser extraída uma regra explicativa difusa por classe, o que prejudica a interpretação das regras em bases de dados com uma grande quantidade de atributos.

A rede EFuNN por sua vez, apresentou resultados significativos na classificação dos padrões de odores, obteve um erro médio de classificação de $0.1 \%$ e $0.88 \%$ para as bases de vinhos e gases, respectivamente. Além da boa performance de generalização, existem outras características que a tornam mais adequada em relação as demais abordagens apresentadas, podem ser destacadas: a não necessidade de pré-processamento nos dados; seu método de aprendizagem incremental e on-line, que permite a rede aprender novos odores durante a operação do nariz artificial; a possibilidade de inserção, extração e agregação de conhecimento, os quais possibilitam que o conhecimento adquirido por um nariz artificial possa ser repassado a outros dispositivos de forma extremamente simples; e a extração de regras de classificação difusas, explicando o conhecimento adquirido pela rede durante $\mathrm{o}$ aprendizado.

Considerando métodos estatísticos não-paramétricos de comparação, com nível de significância de 5\%, as melhores abordagens para a classificação de odores avaliadas neste trabalho foram as redes TDNN utilizando o filtro Wavelet e as redes EFuNN. Futuras pesquisas poderiam considerar outras funcionalidades na construção dos narizes artificiais, como a aquisição automática de conhecimento, configuração automática dos dispositivos, praticidade e a versatilidade dos narizes artificiais.

\section{AGRADECIMENTOS}

Os autores agradecem ao CNPq, CAPES e FINEP (agências brasileiras de fomento à pesquisa) pela ajuda financeira.

\section{REFERÊNCIAS}

Abraham, A. e Nath, B. (2002). Hybrid intelligent systems design: A review of a decade of research, Technical report, Monash University.

Barbosa, M. S. S., Ludermir, T. B., Santos, F. L., Souza, J. E. G., Santos, M. S. e Melo, C. P. (2002). Uma rbf para a classificação de gases da indústria petrolífera, Pro- ceedings do VII Simpósio Brasileiro de Redes Neurais, pp. 130-135.

Brigham, E. (1988). The Fast Fourier Transform and its Applications, Prentice Hall, Englewood Cliffs.

Daubechies, I. (1988). Orthonormal bases of compactly supported wavelets, Communications of Pure and Applied Mathematics 41: 909-996.

Daubechies, I. (1990). The wavelet transform, timefrequency localization and signal analysis, IEEE Transactions on Information Theory 36(5): 961-1005.

Duda, R. O., Hart, P. E. e Stork, D. G. (2001). Pattern Classification, 2 edn, Stork-John Wiley and Sons.

Gardner, J. W., Hines, E. L. e Wilkinson, M. (1990). Application of artificial neural networks to an electronic olfactory system, Meas. Science Technology 1: 446-451.

Gardner, J. W., Pearce, T. C., Friel, S., Bartlett, P. N. e Blair, N. (1994). A multisensor system for beer flavour monitoring using an array of conducting polymers and predictive classifiers, Sensors and Actuators B 18-19: 240243.

Jackson, J. E. (1991). A User's Guide to Principal Components, John Wiley and Sons, Inc., pp. 1-25.

Johson, R. A. e Wichern, D. W. (1999). Applied Multivariate Statistical Analysis, 1 edn, Prentice Hall.

Kasabov, N. (1998). Evolving fuzzy neural networks - algorithms, applications and biological motivation, in $\mathrm{Y}$. T e M. G (eds), Methodologies for the Conception, Design and Application of Soft Computing, World Scientific, pp. 271-274.

Keller, P. E., Kangas, L. J., Liden, L. H., Hashem, S. e Kouzes, R. T. (1995). Electronic noses and their applications, Proceedings of IEEE Technical Applications Conference and Workshop, pp. 116-119.

Keller, P. E., Kouzes, R. T. e Kangas, L. J. (1994). Three neural network based sensor systems for environmental monitoring, Proceedings of IEEE Electro 94 International Conference, pp. 378-382.

Lang, K. J. e Hilton, G. E. (1988). The development of the time-delay neural network architeture for speech recognition, Technical Report CMU-CS-88-152, CarnegieMellon University.

Li, R. P., Mukaidono, M. e Turksen, I. B. (2002). A fuzzy neural network for pattern classification and feature selection, Fuzzy Sets and Systems 130: 101-108. 
Mcgarry, K., Wermter, S. e Maclntyre, J. (2002). Hybrid neural systems: From simple coupling to fully integrated neural networks, Neural Computing Surveys p. 654p.

Medsker, L. R. (1995). Hybrid Intelligent Systems, Kluwer Academic Publishers.

Misiti, M., Misiti, Y., Oppenheim, G. e Poggi, J. M. (1996). Wavelet Toolbox User's Guide for use with Matlab, The MathwWorks Inc., p. 626.

Natale, C. D., Davide, F. A. M., Damico, A., Sberveglieri, G., Nelli, P., Faglia, G. e Perego, C. (1995). Complex chemical pattern recognition with sensor array: the discrimination of vintage years of wine, Sensors and Actuators B 24-25: 801-804.

Persaud, K. C. e Travers, P. J. (1997). Arrays of broad specificity films for sensing volatile chemicals, in E. KressRogers (ed.), Handbook of Biosensors and Electronic Noses: Medicine. Food and the Environment, CRC Press, pp. 563-592.

Prechelt, L. (1994). Proben1 a set of neural network benchmark problems and benchmarking rules, Technical Report 21, Universität Karlsruhe.

Rumelhart, D. E., Hilton, G. E. e Williams, R. J. (1986). Learning representations by backpropagation errors, $\mathrm{Na}$ ture 323: 533-536.

Santos, M. S. (2000). Construção de um Nariz Artificial usando Redes Neurais, Tese (doutorado em ciência da computação), Universidade Federal de Pernambuco, Recife-PE.

Sundgren, H., Winquist, F., Lukkari, I. e Lundström, I. (1991). Artificial neural networks and gas sensor arrays: Quantification of individual components in a gas mixture, Meas. Science Technology 2: 464-469.

Waibel, A., Hanazawa, T., Hinton, G., Shikano, K. e Lang, K. (1989). Phonemic recognition using time delay neural networks, IEEE Trans. Acoust. Speech, Signal Processing 37(3): 328-339.

Yamazaki, A. e Ludermir, T. B. (2001). Classification of vintages of wine by an artificial nose with neural networks, Proceedings of 8th International Conference on Neural Information Processing (ICONIP'2001), Vol. 1, pp. 184-187.

Yamazaki, A., Ludermir, T. B. e Souto, M. C. P. D. (2001). Classification of vintages of wine by an artificial nose using time delay neural networks, IEE Electronics Letters 37(24): 1466-1467.
Zanchettin, C. e Ludermir, T. B. (2003a). Classification of gases from the petroliferous industry by an artificial nose with neural network, Proceedings of Joint 13th International Conference on Artificial Neural Networks and 10th International Conference on Neural Information Processing (ICANN/ICONIP 2003), pp. 208-211.

Zanchettin, C. e Ludermir, T. B. (2003b). A neuro-fuzzy model applied to odor recognition in an artificial nose, Proceedings of Hybrid intelligent System, pp. 917-926.

Zanchettin, C. e Ludermir, T. B. (2003c). Wavelet filter for noise reduction and signal compression in an artificial nose, Proceedings of Hybrid intelligent System, Vol. 88, pp. 907-916. 